\title{
Selective Laser Melting of In Situ TiB/Ti6Al4V Composites: Formability, Microstructure Evolution and Mechanical Performance
}

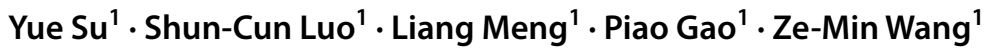 \\ Received: 21 November 2019 / Revised: 6 January 2020 / Published online: 11 March 2020 \\ (c) The Chinese Society for Metals (CSM) and Springer-Verlag GmbH Germany, part of Springer Nature 2020
}

\begin{abstract}
In the present study, a series of in situ TiB/Ti6Al4V composites were fabricated using selective laser melting. The formability, microstructure evolution and mechanical properties of the as-built samples added with different contents of $\mathrm{TiB}_{2}$ were studied. It is found that the densification level is related to both the content of $\mathrm{TiB}_{2}$ and laser energy density. The added $\mathrm{TiB}_{2}$ reinforcement particle can spontaneously react with titanium and then form the TiB phase. The needle-like TiB phase tends to transform into dot-like particles with the decrease in energy density. Additionally, with the increase in $\mathrm{TiB}_{2}$ content, the $\mathrm{TiB}$ phase is coarsened due to the increased nucleation rate and more reactions. The grain morphology is found to largely depend on the translational speed of solid-fluid interface determined by the temperature gradient and cooling rate. Also, the microhardness of the as-built TiB/Ti6Al4V composites is obviously improved. More interestingly, as the energy density increases, the microhardness of the as-built TiB/Ti6Al4V composites firstly increases and then decreases due to the synergy of grain size and different morphologies and distribution of TiB phases. The wear resistance of TiB/Ti6Al4V composites is far superior to that of Ti6Al4V alloy owing to the increased microhardness resulted from the uniform distribution of the hard TiB phase in the matrix.
\end{abstract}

Keywords Selective laser melting $\cdot \mathrm{TiB} / \mathrm{Ti6A14V}$ composites $\cdot$ Energy density $\cdot$ Densification behavior $\cdot$ Microhardness Wear resistance

\section{Introduction}

Owing to their excellent corrosion resistance, high-temperature durability, excellent mechanical properties, titanium and its alloys have been extensively applied in the automobile, aerospace and biological medicine industries [1-4]. However, titanium alloys show poor wear resistance, low hardness, poor high-temperature creep resistance and high production cost, which greatly hinders the application of titanium alloys.

Previous research indicated that the microhardness, wear resistance and high-temperature resistance of titanium and its alloys can be enhanced by adding hard ceramic-reinforced

Available online at http://link.springer.com/journal/40195.

Ze-Min Wang

zmwang@hust.edu.cn

1 Wuhan National Laboratory for Optoelectronics, Huazhong University of Science and Technology, Wuhan 430074, China phases into the matrix [5-8]. Therefore, the ceramic-reinforced titanium matrix composites have attracted more and more attention due to their excellent properties. Among several kinds of reinforced phases applied to produce the ceramic-reinforced titanium matrix composites, TiB is universally used owing to its similar thermal expansion coefficient and stable interface with the matrix [9]. Firstly, boron (B) has good biocompatibility [10], promoting the TiB/titanium alloy matrix composites to be a potential candidate for the medical application. Secondly, the difference of density between the $\operatorname{TiB}\left(4.54 \mathrm{~g} \mathrm{~cm}^{-3}\right)$ and titanium alloys like Ti6Al4V $\left(4.4 \mathrm{~g} \mathrm{~cm}^{-3}\right)$ is relatively small. Thirdly, the composites can be significantly hardened and strengthened by adding only a tiny amount of TiB-reinforced phase [11]. Last but not least, the chemical and thermodynamic stabilities of TiB are good at high temperatures. Meanwhile, the interface between the matrix and the TiB-reinforced phase is stable. The similarity of thermal expansion coefficient between the TiB $\left(7.2 \times 10^{-6}{ }^{\circ} \mathrm{C}^{-1}\right)$ and Ti6Al4V $\left(8.6 \times 10^{-6}{ }^{\circ} \mathrm{C}^{-1}\right)$ reduces the harmful incompatibility, as well as the residual stress of brittle interface. 
In terms of the source of the reinforced phase, the way to prepare ceramic-reinforced metal matrix composites can be defined as the ex situ or in situ synthesis [12-15]. It is practiced that the latter way possesses more superiority, because it avoids the reinforced phase being polluted by an extra addition. The TiB-reinforced phase is formed by an in situ reaction between the $\mathrm{Ti}$ and $\mathrm{TiB}_{2}$, which exhibits a good interfacial bonding between the titanium alloy matrix and TiB-reinforced phase [7]. In this way, the in situ reinforced phase combines with the matrix strongly.

The ceramic-reinforced titanium matrix composites are mostly prepared by traditional processing methods, such as casting [5], arc-melting [16] and so on. In situ synthesized titanium matrix composites reinforced with multiple ceramic particulates including $\mathrm{TiB}, \mathrm{TiC}$ and $\mathrm{Y}_{2} \mathrm{O}_{3}$ were fabricated by $\mathrm{Xu}$ et al. [17] via arc-melting technique utilizing the chemical reaction among $\mathrm{Ti}, \mathrm{B}_{2} \mathrm{O}_{3}, \mathrm{~B}_{4} \mathrm{C}$ and $\mathrm{Y}$. TiB phase grew in needle shape and was distributed uniformly in the titanium matrix. Maseko et al. [18] fabricated Ti-based composites by incorporating $\mathrm{ZrB}_{2}$ to the Ti matrix through spark plasma sintering. The hardness of the composite with $10 \mathrm{wt} \% \mathrm{ZrB}_{2}$ showed the highest hardness $(\sim 595 \mathrm{Hv})$. However, these techniques usually involve time-consuming, energy-consuming and material-consuming production steps. In recent years, new and advanced technology like selective laser melting (SLM), providing rapid manufacturing of complexshaped three-dimensional metal parts with high precision and excellent performance, has great potential in simplifying and accelerating the production process [19]. Additionally, SLM also serves as one of the most popular in situ synthesis methods, perfectly realizing the direct forming process from pre-alloyed powders. During the SLM process, the powder system experiences a process of ultrafast melting and solidification. Meanwhile, complicated thermal cycles take place within molten pools under the irradiation of the laser beam, thus generating fine microstructures [20,21]. Starting from the $\mathrm{Ti}-\mathrm{TiB}_{2}$-milled powders, Hooyar et al. [8] firstly in situ produced nearly fully dense $\mathrm{Ti}-\mathrm{TiB}$ composites using the optimized SLM parameters. Meanwhile, transmission electron microscope (TEM) observation indicated that B significantly contributes to the refinement of Ti grains. The results also indicated that the Vickers microhardness and compression strengths of the Ti-TiB composites obviously increase as compared with the SLM-fabricated pure Ti. However, most studies mentioned above place emphasis on the investigation of Ti-based composites reinforced with TiB particles. Compared to the pure Ti, Ti6Al4V is a dual-phase titanium alloy that combines the advantages of $\alpha$-phase and $\beta$-phase, thus possessing superior comprehensive properties [22, 23].

However, up to now, only a limited number of investigations on the TiB-reinforced Ti6Al4V composites fabricated by SLM have been carried out. Also, the corresponding influences of TiB on cracking and densification behaviors, microstructure evolutions and mechanical properties of the TiB-reinforced Ti6A14V composites remain unclear. As reported earlier, the introduction of the TiB-reinforced phase can result in a significant enhancement of mechanical properties for the primitive material. Hence, this work is aimed to realize fabricating in situ TiB/Ti6Al4V composites added with different contents of $\mathrm{TiB}_{2}$ through SLM at different energy densities. On this basis, the effects of $\mathrm{TiB}_{2}$ content and energy density on formability, microstructure evolution and mechanical properties are studied and discussed in detail. Also, the densification behaviors, phase transformation thermodynamics, TiB forming mechanism and microstructure evolution mechanisms are revealed.

\section{Experimental}

\subsection{Powder Preparation}

A CP titanium plate was chosen as the substrate. Spherical Ti6Al4V powders (Fig. 1a) possessing a mean particle size of $29 \mu \mathrm{m}$ were chosen as the raw material, and the $\mathrm{TiB}_{2}$ powders (Fig. 1b) with the particle sizes about 3-5 $\mu \mathrm{m}$ and purity of $99.9 \%$ were chosen as the reinforcing additives. Before mixing, both powders were dried in a vacuum drying oven at $100{ }^{\circ} \mathrm{C}$ to improve the flowability. Then, the mixture consisting of Ti6Al4V powders and $\mathrm{TiB}_{2}$ powders with varying weight percentages $(0 \mathrm{wt} \%, 1 \mathrm{wt} \%$ and $3 \mathrm{wt} \%)$ were homogenously blended for $6 \mathrm{~h}$ in a low-energy ball mill. As the mixing process was finished, the $\mathrm{TiB}_{2}$ particles were found to disperse uniformly in the powder mixture, as shown in Fig. 1c, d.

\subsection{SLM Process}

A series of specimens were produced via a self-developed SLM system under a protective argon (Ar) atmosphere. These specimens were built at a laser power $(P)$ of $200 \mathrm{~W}$, layer thickness $(t)$ of $0.02 \mathrm{~mm}$ and hatching space $(h)$ of $0.08 \mathrm{~mm}$. To investigate the variation of microstructures and mechanical properties with energy density for the TiB/Ti6Al4V composites added with different contents of $\mathrm{TiB}_{2}$ [24], the laser scanning speed ( $v$ ) was set from 300 to $1200 \mathrm{~mm} / \mathrm{s}$ with an interval of $100 \mathrm{~mm} / \mathrm{s}$. The volumetric energy densities $(E)$ of $416.67,312.5,250,208.33,178.57$, $156.25,138.89,125,113.64$ and $104.17 \mathrm{~J} / \mathrm{mm}^{3}$, respectively, correspond to the selected speeds above, which can be calculated from:

$E=\frac{P}{v \cdot h \cdot t}$. 

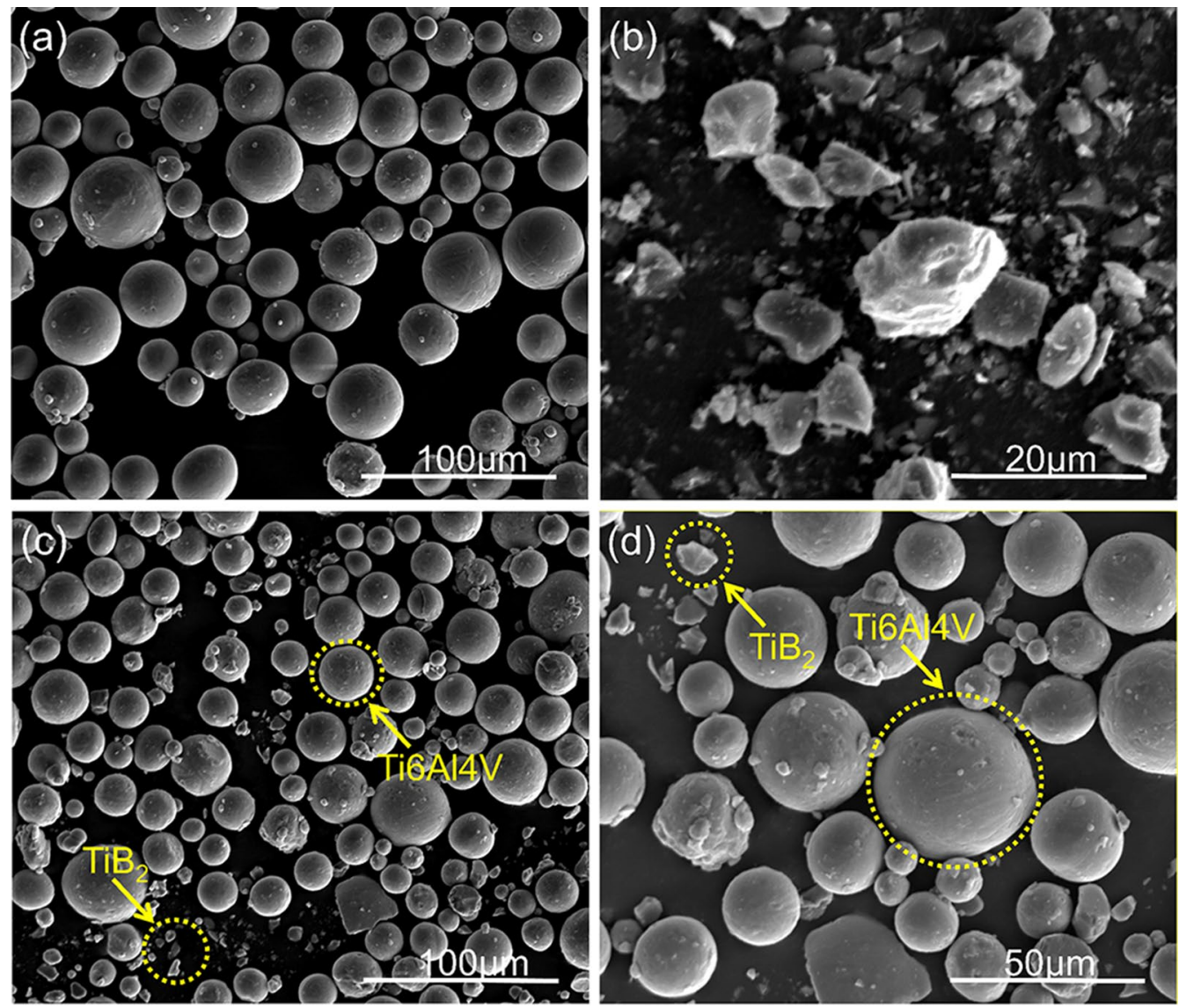

Fig. 1 SEM micrographs showing the morphologies of a Ti6Al4V powders; $\mathbf{b} \mathrm{TiB}_{2}$ powders; $\mathbf{c} \mathrm{Ti} 6 \mathrm{Al} 4 \mathrm{~V}+3$ wt $\%$ TiB 2 powders; $\mathbf{d}$ a magnification figure of $\mathbf{c}$

A simple linear raster scanning pattern was utilized, and the direction of laser scanning changed with an angle of $90^{\circ}$ for each finished layer. Therefore, three groups of specimens fabricated using different process parameters were used to investigate the impacts of the reinforcement content and volumetric energy density on the microstructure evolutions and mechanical properties of TiB/Ti6Al4V composites.

\subsection{Microstructure Characterization}

To prepare the metallographic specimens for further examination, the as-built specimens were cut, ground and finally polished following standard procedures. Phase identification was conducted via an X-ray diffractometer (XRD, PANalytical X'Pert PRO) with $\mathrm{Cu} K_{\alpha}$ radiation. The transverse and longitudinal morphologies, as well as the densification behaviors of the as-built samples, were observed using a Nikon Epiphot 300 optical microscope (OM). The relative densities of the as-built specimens were determined according to the porosities which were calculated by the measuring software of Image-Pro Plus. For the microstructure observation, the polished specimens were etched with Kroll reagent (2\% $\mathrm{HF}, 8 \% \mathrm{HNO}_{3}$ and $90 \%$ $\mathrm{H}_{2} \mathrm{O}$ ) for $50 \mathrm{~s}$ at room temperature and then observed via a scanning electron microscope (SEM) which is equipped with an energy-dispersive spectrometer (EDS). The chemical compositions and element distributions were detected via EDS analysis.

\subsection{Characterization of Mechanical Properties}

The Vickers microhardness was obtained using an HVS1000 tester under a load of $500 \mathrm{~g}$ and a dwell time of $20 \mathrm{~s}$. The friction properties were characterized by the dry sliding wear tests via a ball-on-disk tribometer at room temperature. During the tests, a $\mathrm{Si}_{3} \mathrm{~N}_{4}$ ball with a diameter of $6.35 \mathrm{~mm}$ slid against the flat surface repeatedly without lubrication. For each test, the sliding distance was set as $8 \mathrm{~mm}$, with a sliding speed of $10 \mathrm{~mm} / \mathrm{s}$, a load of $60 \mathrm{~N}$ and a total sliding time of $60 \mathrm{~min}$. The values of the coefficient of friction (COF) were dynamically calculated and recorded by a computer. 


\section{Results and Discussion}

\subsection{Formability and Cracks}

Figure 2 lists a process window of the as-built samples (with $0 \mathrm{wt} \%, 1 \mathrm{wt} \%$ and $3 \mathrm{wt} \% \mathrm{TiB}_{2}$ added, respectively) at all applied energy densities. All the as-built specimens are separated into two parts according to the formability. The blue area represents the well-formed samples, while in the red area, the samples are cracked or even break into pieces. As the content of $\mathrm{TiB}_{2}$ varies, the values of energy density under which the samples cannot be well formed differ. Those $\mathrm{TiB}_{2}$-free specimens are formed with a favorable appearance until the energy density is decreased to $113.64 \mathrm{~J} / \mathrm{mm}^{3}$. Cracks in the samples with $1 \mathrm{wt} \% \mathrm{TiB}_{2}$ are first observed at an energy density of $208.33 \mathrm{~J} / \mathrm{mm}^{3}$. However, the cracks in the samples with $3 \mathrm{wt} \% \mathrm{TiB}_{2}$ primarily occur when the energy density is $312.5 \mathrm{~J} / \mathrm{mm}^{3}$. As can be observed from Fig. $2 \mathrm{a}$, the $\mathrm{TiB}_{2}$-free samples possess better formability. In other words, with the addition of ceramic-reinforced phases, the formability of composites is impaired. Figure 2b, c shows the surface appearance of samples with cracks. It is noteworthy that the upper surface of both samples is smooth without any obvious cracks, while the cracks apparently exist on the longitudinal surface. They extend along the deposited layers, almost perpendicular to the build direction. Preliminarily, it can be concluded that the cracks occur between the deposited layers and mainly extend along the deposited layers, exhibiting a noteworthy orientation.
Figure 3 shows the representative longitudinal $\mathrm{OM}$ and SEM micrographs of cracks in the as-built samples with $3 \mathrm{wt} \% \mathrm{TiB}_{2}$. From Fig. 3a, b, it is obvious that the cracks originate from the edge and end in the interior of the sample or even run through the sample. Figure $3 \mathrm{c}-\mathrm{e}$ shows the partially enlarged SEM micrographs at the edge of the sample. Obviously, the crack always starts from the rugged side wall which contacts directly with the powder bed. As the samples are built by melting powders layer by layer, many semi-molten and unmelted powders are absorbed when the liquid metal touches with the powder bed. Consequently, tiny cracks are prone to generate and thus the strength of the side wall is weakened. Moreover, the edges of the sample suffer from less constraint, which also creates a condition for the formation of cracks. Similar cracking mechanism has also been illustrated in Ref. [25]. Figure 3d shows a short length of the crack near the end. The arc-shaped terminal is generated to avoid stress concentration, thus contributing to the termination of the crack.

To further analyze the cracking behavior, the SEM morphology of the side wall of broken pieces from an as-built sample is shown in Fig. 4. It can be observed that many scattered and accumulated pellets are embedded in the cross section of the crack, as marked by yellow dotted circles. Pits indicated by yellow arrows are formed as a result of the peeling of small pellets embedded in the matrix. The reason for the formation of pellets may be that the overhigh laser energy input results in the decrease in the surface tension of molten pools. Consequently, the splashing of droplets from the surface of the molten pool occurs. The inlaid pellets are easy to flake off due to their low bonding strength with

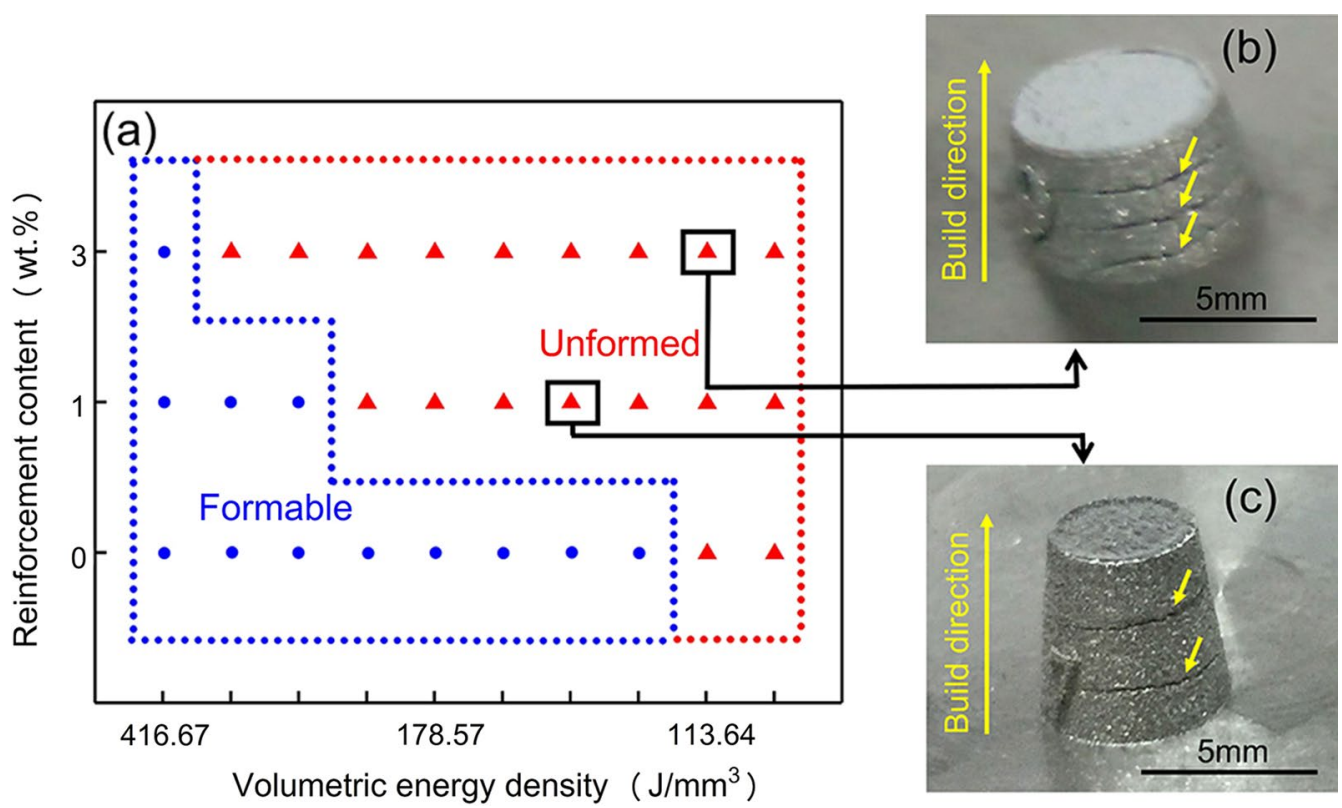

Fig. 2 a A process window; b, c surface appearance of the as-built samples 


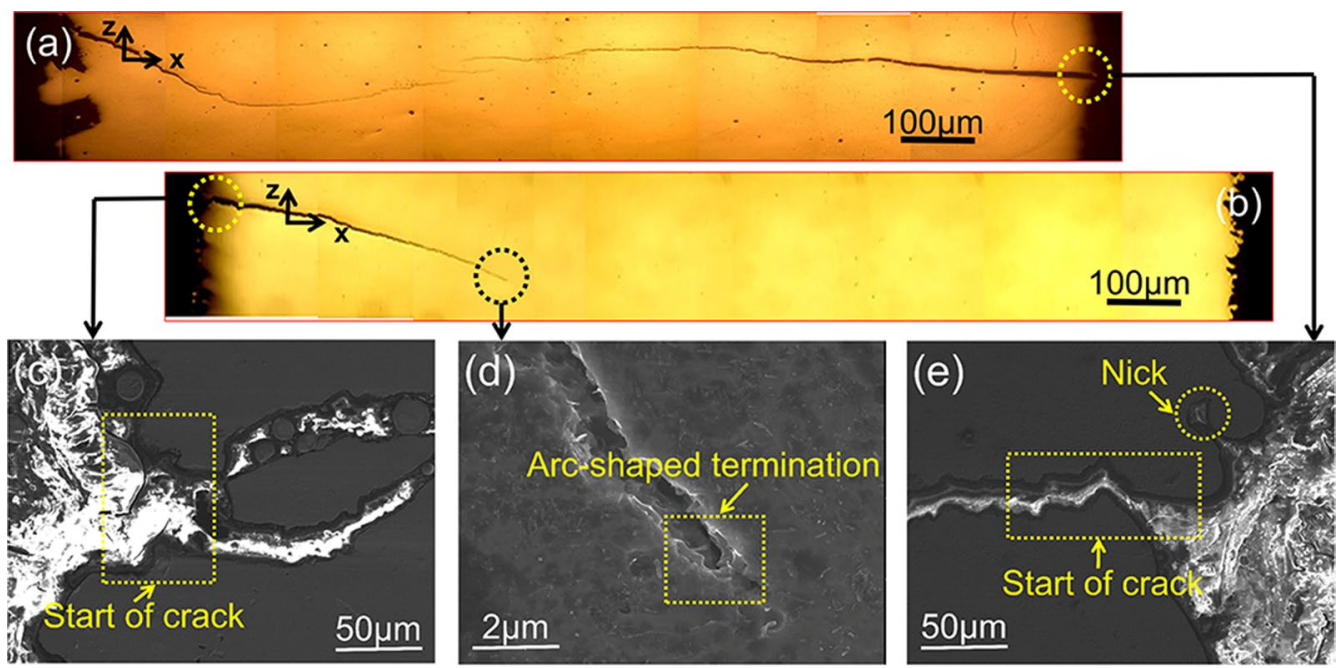

Fig. $3 \mathrm{OM}$ and SEM micrographs of cracks in the as-built samples with $3 \mathrm{wt} \% \mathrm{TiB}_{2}$ on the longitudinal section at varied energy densities: a, e $156.25 \mathrm{~J} / \mathrm{mm}^{3} ; \mathbf{b}-\mathbf{d} 104.17 \mathrm{~J} / \mathrm{mm}^{3}$

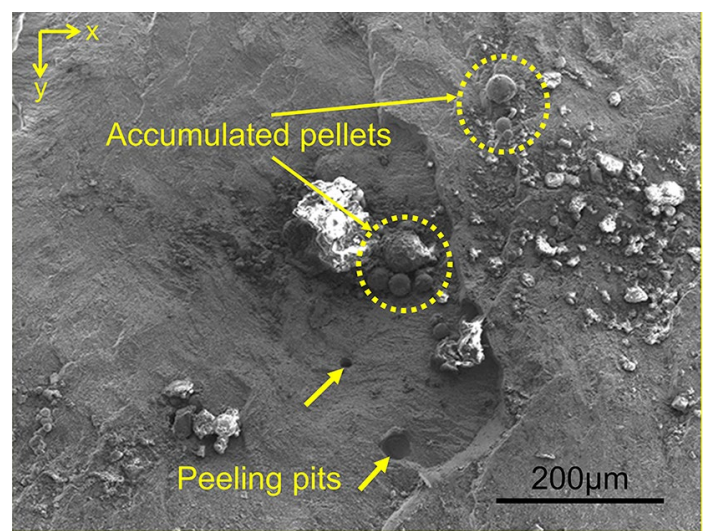

Fig. 4 SEM micrograph displaying the morphology of side wall of the cracked position

the matrix. These factors, however, associated with the tiny cracks on the side wall, are liable to cause cracking.

\subsection{Densification Behavior}

Figure 5 shows the optical micrographs of samples and the variation of density with the energy densities for the TiB/Ti6Al4V composites added with different contents of $\mathrm{TiB}_{2}$. As increasing the energy density from 104.17 to $416.67 \mathrm{~J} / \mathrm{mm}^{3}$, the densities of $1 \mathrm{wt} \% \mathrm{TiB} / \mathrm{Ti} 6 \mathrm{Al} 4 \mathrm{~V}$ composites decrease from 99.937 to $98.449 \%$ monotonously, and a decrease in density from 99.325 to $97.272 \%$ is also observed in $3 \mathrm{wt} \% \mathrm{TiB} / \mathrm{Ti} 6 \mathrm{Al} 4 \mathrm{~V}$ composites. However, the densities of Ti6Al4V samples exhibit a contrary trend as the increase in density from 80.42 to $99.884 \%$. At the same applied energy density, the density of $1 \mathrm{wt} \%$ TiB/Ti6Al4V composite is higher than that of $3 \mathrm{wt} \% \mathrm{TiB} / \mathrm{Ti} 6 \mathrm{Al} 4 \mathrm{~V}$ composite. Actually, similar results of a decrease in density with the addition of ceramic reinforcement have been reported before [26]. Generally, the formation mechanisms for the voids with different morphologies are different. The insufficient melting of powder particles and instabilities of molten pools will lead to the formation of unmelted particles and irregular voids [27], whereas the spherical voids are generally reported as the result of keyhole effect induced by the excess energy input [28].

As for the Ti6Al4V samples, the formation of the irregular voids under low-energy densities (high laser scanning speeds) is ascribed to insufficient energy input. At a relatively low-energy density, the inputted laser energy is too low to fully fuse the powders. The molten pools will be disturbed or even interrupted by the unmelted particles, leading to the formation of discontinuous molten pools. As a result, the interspaces between powders and the pores between layers are difficult to be filled completely, hence causing irregular voids and lower density. The previous study has proved that the addition of $\mathrm{TiB}_{2}$ ceramic reinforcement particles can enhance the interactions between the laser beam and powder particles and significantly improve the absorption irradiance of laser for the powder bed [29]. As for the TiB/ Ti6Al4V composites, the absorptivity of powders for the laser is increased due to the addition of $\mathrm{TiB}_{2}$ particles. As compared to other constituents, aluminum has a lower boiling point of $\sim 2400{ }^{\circ} \mathrm{C}$, below the maximum temperature that occurs during SLM, which is reported up to about $3200{ }^{\circ} \mathrm{C}$ [30]. With more addition of $\mathrm{TiB}_{2}$, the excess energy input (low laser scanning speed) may cause more severe evaporation of $\mathrm{Al}$ and thus the keyhole effect, leading to lower densities on composites. Therefore, the presence of spherical 


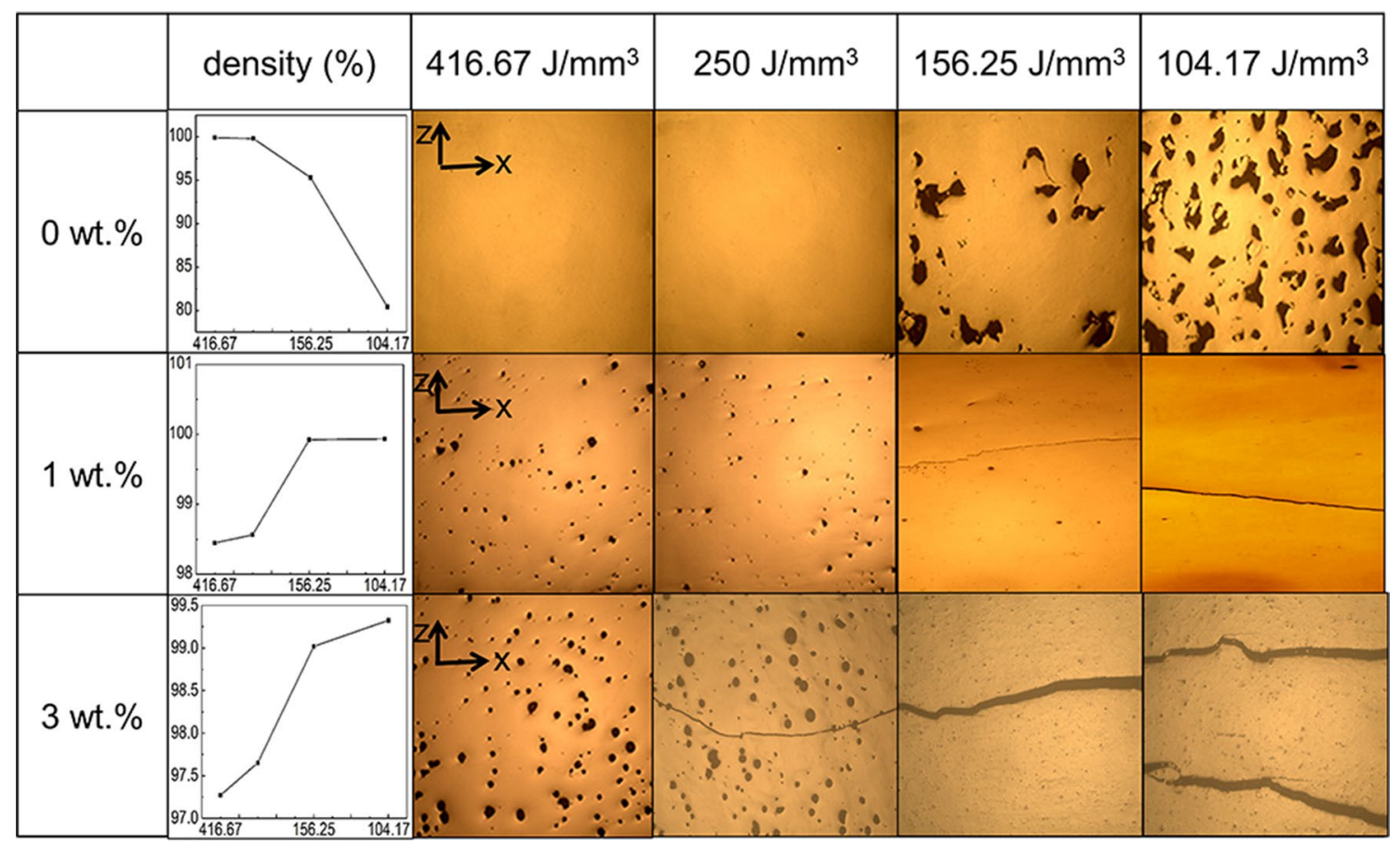

Fig. 5 Line graphs showing the variation of density with the content of $\mathrm{TiB}_{2}$ and optical micrographs showing the appearance of pores and cracks at different energy densities

voids within the TiB/Ti6Al4V composites is correlated with the excess energy input, which induces the evaporation of constituents of the TiB/Ti6Al4V composites with low boiling points. Attar et al. [8] also obtained similar conclusion by investigating the densification behaviors of the SLMed $\mathrm{TiB} / \mathrm{Ti}$ composites.

\subsection{Phase Transformation Mechanism and Identification}

Instead of a reaction between $\mathrm{Ti}$ and $\mathrm{ZrB}_{2}$ in Ref. [18] or $\mathrm{B}_{4} \mathrm{C}$ in Ref. [31], the metal-ceramic composites like TiB/ Ti6Al4V in this study were produced by an in situ reaction between the molten $\mathrm{Ti}$ and reacting $\mathrm{TiB}_{2}$. Because the melting point of $\mathrm{TiB}_{2}$ is higher than that of $\mathrm{Ti}$, once the temperature reaches the melting point of $\mathrm{Ti}$, the uniformly distributed $\mathrm{TiB}_{2}$ particles will react with the molten Ti subsequently. In the activated state, the following reaction will happen:

$\mathrm{Ti}+\mathrm{TiB}_{2} \rightarrow 2 \mathrm{TiB}$.

Based on the thermodynamic data [32], the standard free energy for the above reaction can be described as Eq. (3-3). The variation trend of Gibbs free energy $(\Delta G)$ with the temperature can be drawn by calculation, as shown in Fig. 6. It can be observed that all the values of $\Delta G$ at the temperature ranging from 200 to $1500 \mathrm{~K}$ are negative, which indicates

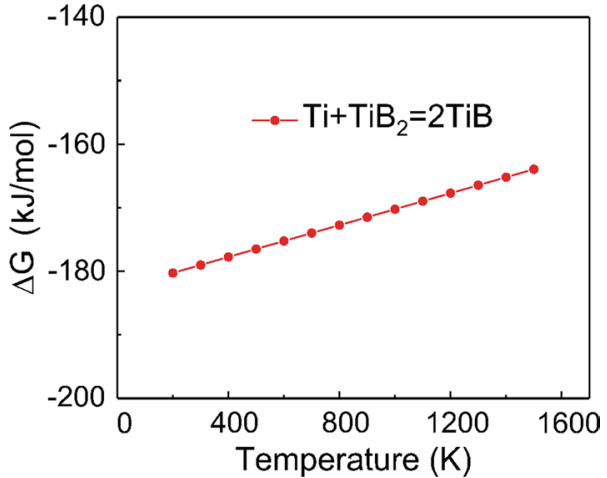

Fig. 6 Variation of Gibbs free energy $\Delta G$ with temperature

that the reaction can spontaneously take place during the SLM process.

$\Delta G=-182765+12.55 T \mathrm{~J} / \mathrm{mol}$.

According to the Ti-B binary phase diagram [33], an appropriate section is adopted, as demonstrated in Fig. 7 (the red double arrow line represents the present composition in this work). The solidification path and phase transformation process are illustrated as follows:

$\mathrm{L} \rightarrow \mathrm{L}+\beta-\mathrm{Ti} \rightarrow \beta-\mathrm{Ti} \rightarrow \alpha-\mathrm{Ti}+\beta-\mathrm{Ti}+\mathrm{TiB}$. 


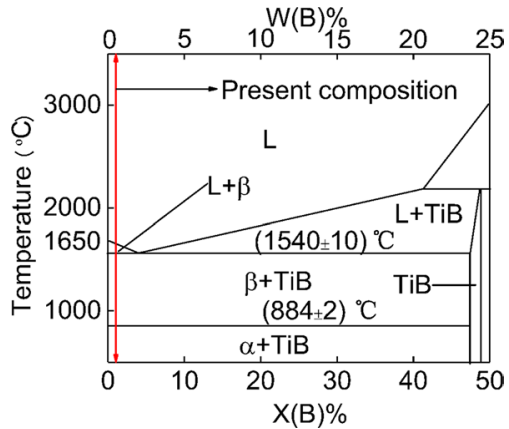

Fig. 7 Partial section of the Ti-B binary phase diagram

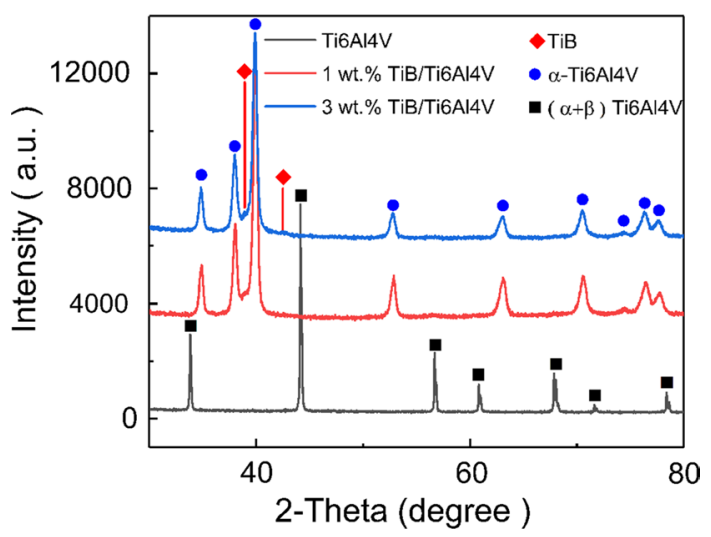

Fig. 8 XRD patterns of the as-built TiB/Ti6Al4V composites with different contents of $\mathrm{TiB}_{2}$
It can be seen from the solidification path that solidification first takes place with the formation of the primary $\beta$-Ti phase. Then an eutectic mixture of $\beta$-Ti and TiB phase is generated from the primary $\beta$-Ti phase. As the temperature falls, $\beta$-Ti begins to transform into another allotropy of $\alpha$-Ti. Due to the tiny addition of $\mathrm{TiB}_{2}$ in this study, once the $\mathrm{B}$ solute is rejected into the solidification front, it can diffuse readily into the remaining liquid metal. Thus, only when the $\mathrm{B}$ solute is saturated in the liquid of the interdendritic region, $\mathrm{TiB}$ will precipitate out. That is to say, the formation of $\beta$-Ti takes place more readily. When the new solid $\beta$-Ti forms, the $B$ solute will be gradually accumulated in the interdendritic region, which results in the formation of TiB. As a consequence, the reduction in $\mathrm{B}$ solute reduces the constitutional supercooling and inhibits the nucleation of TiB crystals.

To confirm the composition of phases that exist in the samples, XRD analysis was performed. The XRD patterns of the as-built specimens are depicted in Fig. 8. The main diffraction peaks correspond to Ti6Al4V $(\alpha)$ and TiB phases. The detected diffraction peaks of the TiB phase are not evident owing to its tiny content.

To investigate the presence of the TiB phase in the asbuilt TiB/Ti6Al4V composites in detail, the composition distribution of a partial transverse section of the sample added with $1 \mathrm{wt} \% \mathrm{TiB}_{2}$ is analyzed by EDS, as shown in Fig. 9. The whole observed view is dominated by the graycolor matrix and the white-color precipitates with a basket shape. It is a remarkable fact that very clear phase boundaries appear due to the precipitation of the white-color phase. The result of EDS analysis discloses that the white-color precipitate is rich in $\mathrm{B}$ and $\mathrm{V}$ while the gray-color matrix is enriched in $\mathrm{Ti}, \mathrm{Al}$ and $\mathrm{V}$ elements. Therefore, combined
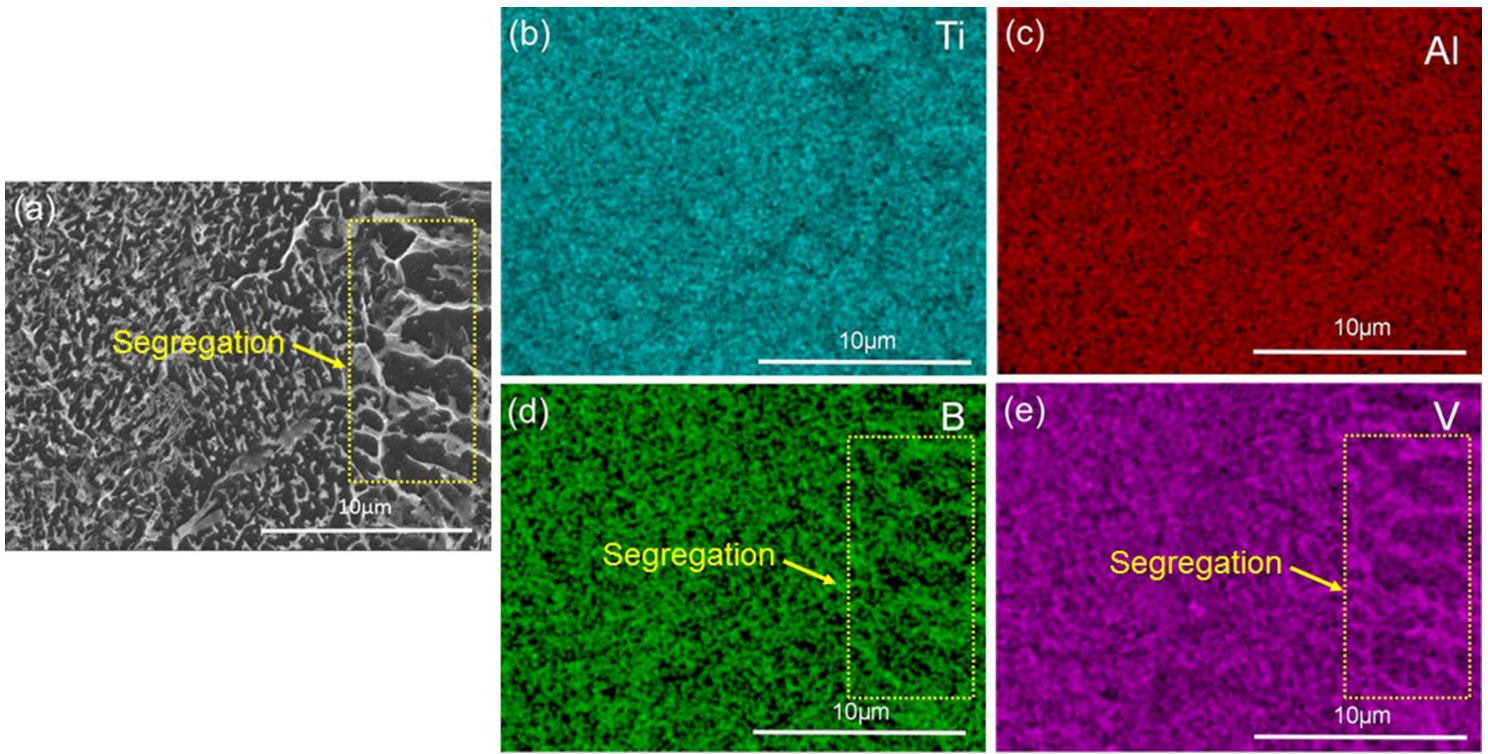

Fig. 9 EDS images showing the cross section of the as-built TiB/Ti6Al4V composites 
with the XRD patterns, it can be deduced that the whitecolor precipitate is $\mathrm{TiB}$ and the gray-color matrix refers to Ti6Al4V. Interestingly, the segregation of V element occurs in the region where $\mathrm{TiB}$ precipitates out. $\mathrm{TiB}$ is in situ synthesized via the reaction of $\mathrm{Ti}$ and $\mathrm{TiB}_{2}$, which consumes the Ti solute from the Ti6Al4V matrix. Generally, $\alpha-\mathrm{Ti}$ and $\beta$-Ti are mainly distributed in the Ti6Al4V matrix and grain boundaries, respectively $[34,35]$. Also, $\mathrm{Al}$ element is relatively stable in $\alpha$ phase, while the $\mathrm{V}$ element is stable in $\beta$ phase. When the in situ reaction occurs, the remained $\mathrm{Al}$ in the matrix starts to diffuse to maintain the homogeneity of $\mathrm{Al}$ in the whole structure, while $\mathrm{V}$ is retained at the grain boundaries due to its high stability in $\beta$ phase. As a result, the content of $\mathrm{V}$ at the grain boundaries is higher than that of $\mathrm{Al}$, generating obvious segregation of $\mathrm{V}$.

\subsection{Microstructure Evolution}

\subsubsection{Effect of Reinforcement Content}

Figure 10 shows the SEM micrographs on the cross and longitudinal sections of the as-built TiB/Ti6Al4V composites with $1 \mathrm{wt} \%$ and $3 \mathrm{wt} \% \mathrm{TiB}_{2}$ at the energy density of $416.67 \mathrm{~J} / \mathrm{mm}^{3}$. As described above, the TiB phase is evenly distributed in the Ti6Al4V matrix and mostly grows along the grain boundaries (as demonstrated in Fig. 10a-d), thus exhibiting a phenomenon of boundary segregation. In terms of the periodic bond chain theory [36], the TiB phase prefers to grow along the [010] direction rather than along the [100], [101] and [001] ones. This mechanism is proved by the study in Ref. [8] in which TiB-reinforced titanium matrix composites were produced. As a consequence, needle-like TiB phase with B27 structure precipitates out with the needle axis paralleling to the [010] orientation, as demonstrated in Fig. 10e-h. The in situ synthesized TiB phase tends to nucleate and grow on the planes with matched lattice, in which the lattice strain existed at the interface between the $\mathrm{TiB}$ and matrix is minimal. Thus, the similarity of the lattice structures between the TiB and $\beta$-Ti facilitates the nucleation and growth of TiB needles. As a result, the TiB phase is adjacent to $\beta$-Ti and extends into the $\alpha$-Ti matrix in a particular direction. This agrees well with the observed results shown in Fig. 10a-d, and some TiB needles extend from the boundaries into the interior of the matrix grains.

Additionally, it can be obviously observed that the content of $\mathrm{TiB}_{2}$ has an effect on the size of matrix grains and $\mathrm{TiB}$. The matrix grains added with more $\mathrm{TiB}_{2}$ are finer in comparison with those with less $\mathrm{TiB}_{2}$. Because $\mathrm{TiB}$ precipitates out within the matrix through the reaction between $\mathrm{Ti}$ and $\mathrm{TiB}_{2}$, when the nucleated crystals come into contact with each other, they will stop growing and form the boundaries [37, 38]. Because the boundaries are easier to form with more addition of $\mathrm{TiB}_{2}$, the matrix grains of $3 \mathrm{wt} \% \mathrm{TiB} / \mathrm{Ti} 6 \mathrm{Al} 4 \mathrm{~V}$ are finer. However, as for the $\mathrm{TiB}$ needles, with increasing content of $\mathrm{TiB}_{2}$, the nucleation rate is increased and more reactions will occur at the same time, leading to the coarsening of TiB needles (Fig. 10d).

\subsubsection{Effect of Energy Density}

Figure 11 shows the microstructure evolution of the as-built $\mathrm{TiB} / \mathrm{Ti} 6 \mathrm{Al} 4 \mathrm{~V}$ composites with $1 \mathrm{wt} \% \mathrm{TiB}_{2}$ on the cross section at different energy densities. Noticeably, two zones with varied grain sizes appear, as shown in Fig. 11a-d. The two zones are, respectively, defined as zone I with fine equiaxed crystals and zone II with coarse equiaxed crystals. When the energy density is decreased from 416.67 to $104.17 \mathrm{~J} / \mathrm{mm}^{3}$, the grains of the two zones are simultaneously refined. Meanwhile, the space of zone II is gradually narrowed, as indicated in Fig. 11d. During SLM, a moving heat source with a mode of Gaussian distribution is applied, which can be expressed as [39]:

$q=\frac{2 A P}{\pi R^{2}} \exp \left(-\frac{2 r^{2}}{R^{2}}\right)$

where $A$ denotes the energy absorptivity of powder particles, which is largely related to the laser wavelength, surface conditions and physical properties of the powder particles. $P$ denotes the laser power, $R$ denotes the effective radius of laser beam where the energy density decreases to $1 / e^{2}$ in the center of the laser spot, $r$ denotes the radial distance from a random point to the center of the laser spot. During the SLM process, the powders are partially fused by the moving laser source, thus forming the micro-molten pools. The molten metal in these pools experiences fast cooling and solidification. The morphology of grains largely depends on the solidification condition, including the temperature gradient and translational speed of the solid-fluid interface. The temperature gradient can be expressed as [40]:

$G=\sqrt{G_{x}^{2}+G_{y}^{2}+G_{z}^{2}}$,

where $G_{x}, G_{y}$ and $G_{z}$ are the temperature gradients along the directions of $x, y$ and $z$, correspondingly. The cooling rate of molten metal in the molten pools can be expressed as [40]:

$V=\left|\frac{\mathrm{d} T}{\mathrm{~d} t}\right|$.

Thus, the translational speed of the solid-fluid interface can be denoted as [40]:

$R=\frac{V}{G}=\frac{\left|\frac{\mathrm{d} T}{\mathrm{~d} t}\right|}{\sqrt{G_{x}^{2}+G_{y}^{2}+G_{z}^{2}}}$.

Obviously, the heat source with a Gaussian mode causes a different distribution of temperature in the melting tracks. 

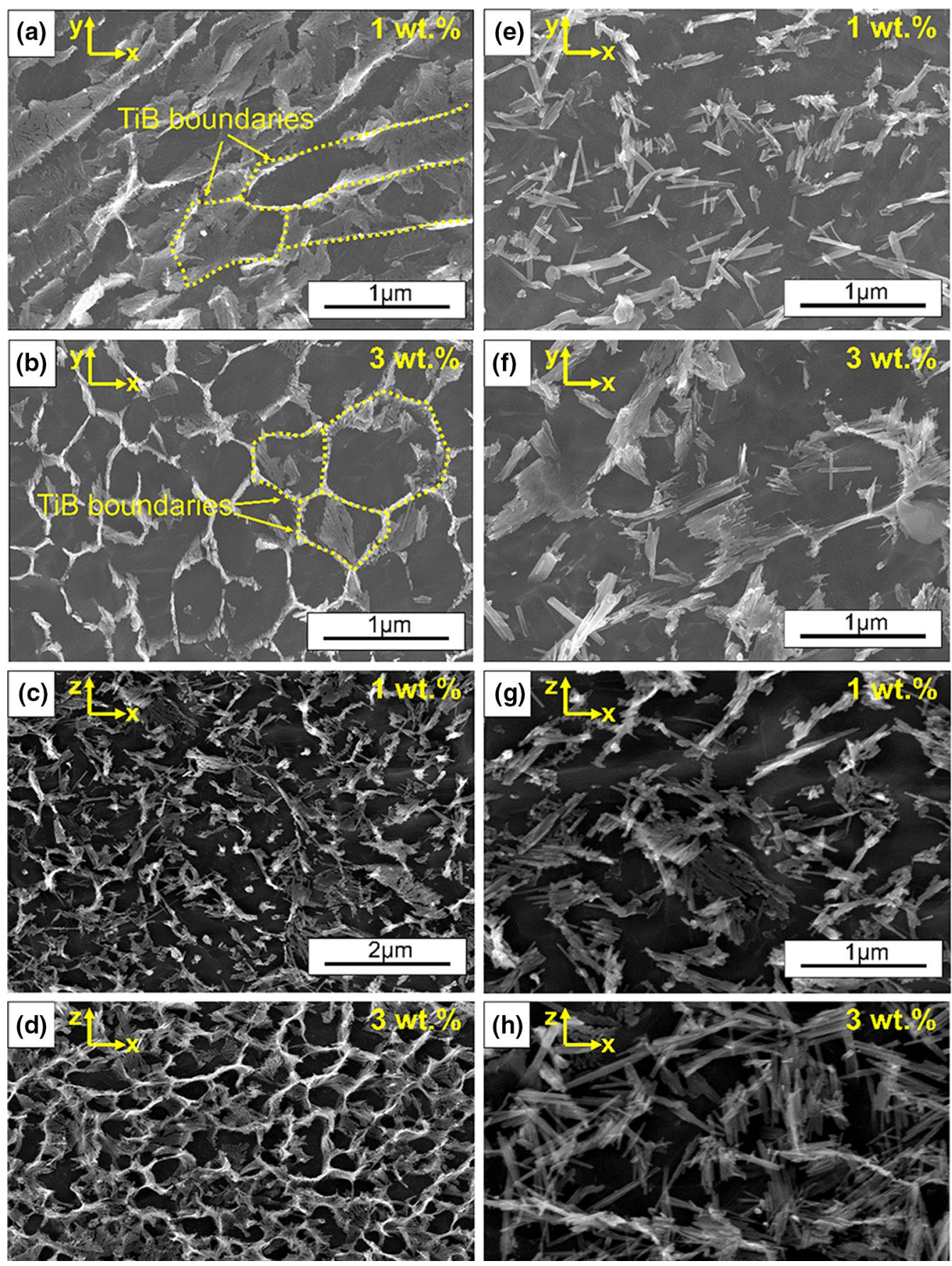

Fig. 10 SEM micrographs on the cross and longitudinal sections displaying the morphologies of matrix and TiB needles for the as-built samples at the energy density of $416.67 \mathrm{~J} / \mathrm{mm}^{3}$ : a, e $1 \mathrm{wt} \%$, cross section; $\mathbf{b}, \mathbf{f} 3 \mathrm{wt} \%$, cross section; c, $\mathbf{g} 1 \mathrm{wt} \%$, longitudinal section; d, h $3 \mathrm{wt} \%$, longitudinal section

Also, the fast-moving laser beam leads to a high cooling rate. Both factors contribute to the high-temperature gradient and different cooling rates inside the melting tracks. According to the equations mentioned above, the growth rates of grains at different zones in the melting tracks differ. The grains within the center region of the laser spot absorb more energy, thus generating smaller temperature gradient and lower cooling rates. As a consequence, the grains in the center possess more ability to grow up and are prone to be coarsened. As the centrifugal distance increases, the temperature gradient and cooling rate increase, thus the grains become finer. That is the reason why two different zones exist. The energy density affects the grain size as the interaction time differs. As the energy density decreases, 

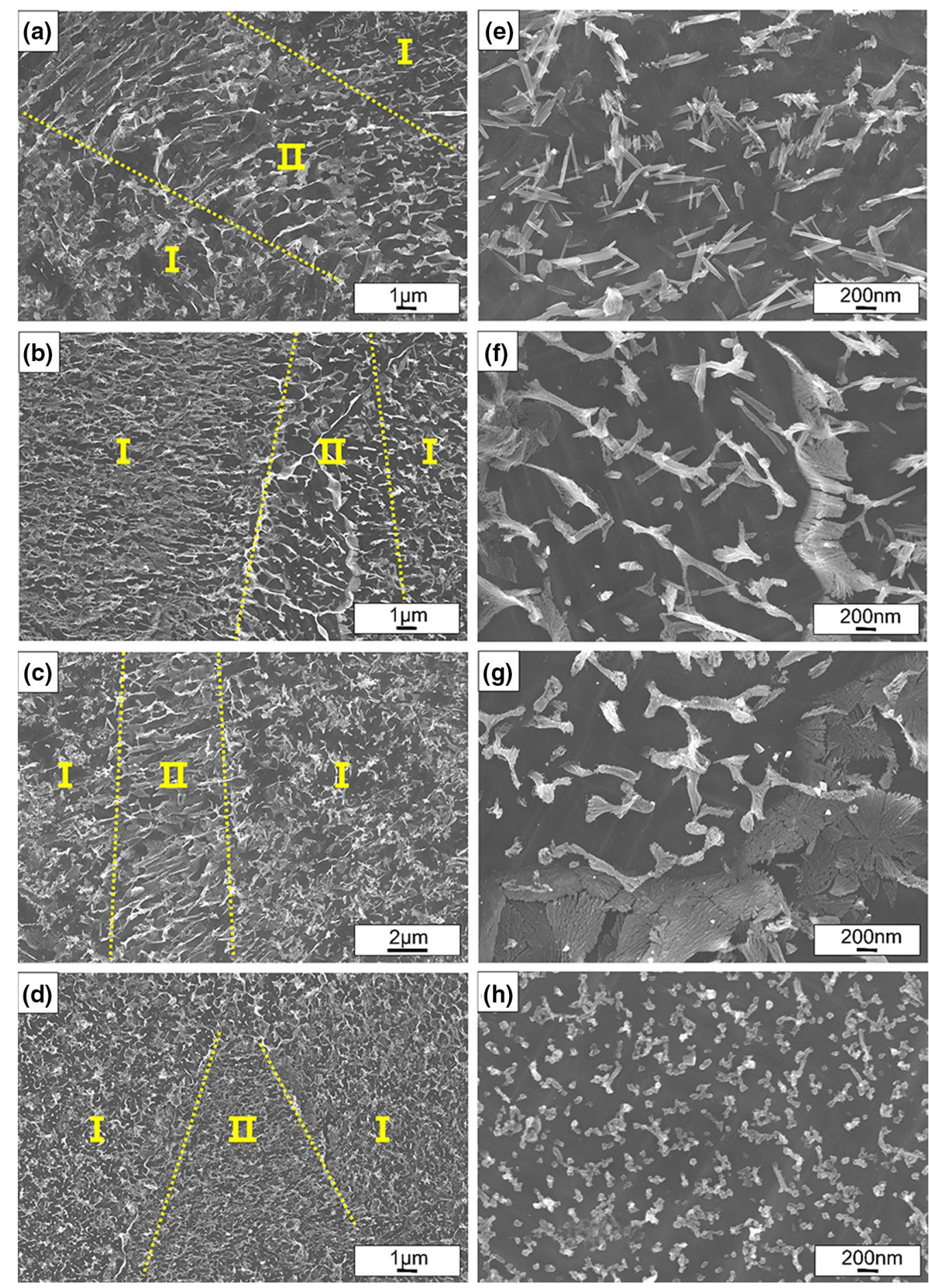

Fig. 11 SEM micrographs on the cross section showing the microstructures of the as-built samples with 1 wt $\%$ TiB ${ }_{2}$ and the morphology of TiB phase at varied energy densities: a, e $416.67 \mathrm{~J} / \mathrm{mm}^{3} ; \mathbf{b}, \mathbf{f} 250 \mathrm{~J} / \mathrm{mm}^{3} ; \mathbf{c}, \mathbf{g} 156.25 \mathrm{~J} / \mathrm{mm}^{3} ; \mathbf{d}, \mathbf{h} 104.17 \mathrm{~J} / \mathrm{mm}^{3}$

the interaction time between the laser heat source and powder bed is shortened. Consequently, both the temperature gradient and the cooling rate increase. Because the effect of the cooling rate is stronger than that of the temperature gradient, the grains grow faster and become finer. As the energy density is reduced to $104.17 \mathrm{~J} / \mathrm{mm}^{3}$, the affected area of the laser beam is shrunk and the difference among the two zones becomes unapparent. It is noteworthy that the microstructure becomes more and more non-uniform with the decrease in energy density. This is because the TiB 
crystals have no enough time to nucleate before the molten metal solidifies. Also, the asynchrony of precipitation leads to apparent segregation, which results in the inhomogeneity of microstructure.

As for the TiB phase, it becomes finer with the energy density decreasing from 416.67 to $104.17 \mathrm{~J} / \mathrm{mm}^{3}$, as depicted in Fig. 11e-h. Meanwhile, the morphology changes from needles to particle shape. At a high energy density, the TiB phase almost fully precipitates out and exhibits preferential growth along the [010] direction, thus forming the needlelike TiB. Inversely, the decrease in energy density gives rise to the decline of chemical reaction time as well as solidification time, which is unfavorable for $\mathrm{TiB}$ to precipitate out continuously. Thus, TiB particles are formed, as demonstrated in Fig. 11h.

Additionally, to better study the evolution of microstructure of the longitudinal section, the columnar crystals existed in the molten pools are observed by SEM, as shown in Fig. 12. As the energy density changes from a high value of $416.67 \mathrm{~J} / \mathrm{mm}^{3}$ to a low one of $104.17 \mathrm{~J} /$ $\mathrm{mm}^{3}$, the columnar crystals are significantly refined. As described above, the grain growth is mainly affected by the temperature gradient and cooling rate. The former determines the grain growth orientation and the latter determines the grain size. Based on the crystallization theory [41], each crystal structure chooses its preferred orientation where it can grow with the highest rate. However, once the preferred crystal orientation is different from that of the highest temperature gradient, the preferred growth of grains will be inhibited, thus generating the cellular dendrites or tiny columnar crystals. As the energy density decreases, the cooling process of the whole molten pool is accelerated and the high cooling velocity will prevent the grains from growing continuously along the preferred crystal orientation. As a result, the coarse columnar crystals are refined and gradually transform into the cellular dendrites, as shown in Fig. 12a, b. When the inputted energy is relatively low like $104.17 \mathrm{~J} / \mathrm{mm}^{3}$ in this study, the extremely high cooling rate makes the columnar crystals almost disappear, as demonstrated in Fig. 12d. Also, the precipitated $\mathrm{TiB}$ needles transform into fine particles with the decrease in energy density, which agrees well with the evolution rule of microstructures on the cross section.
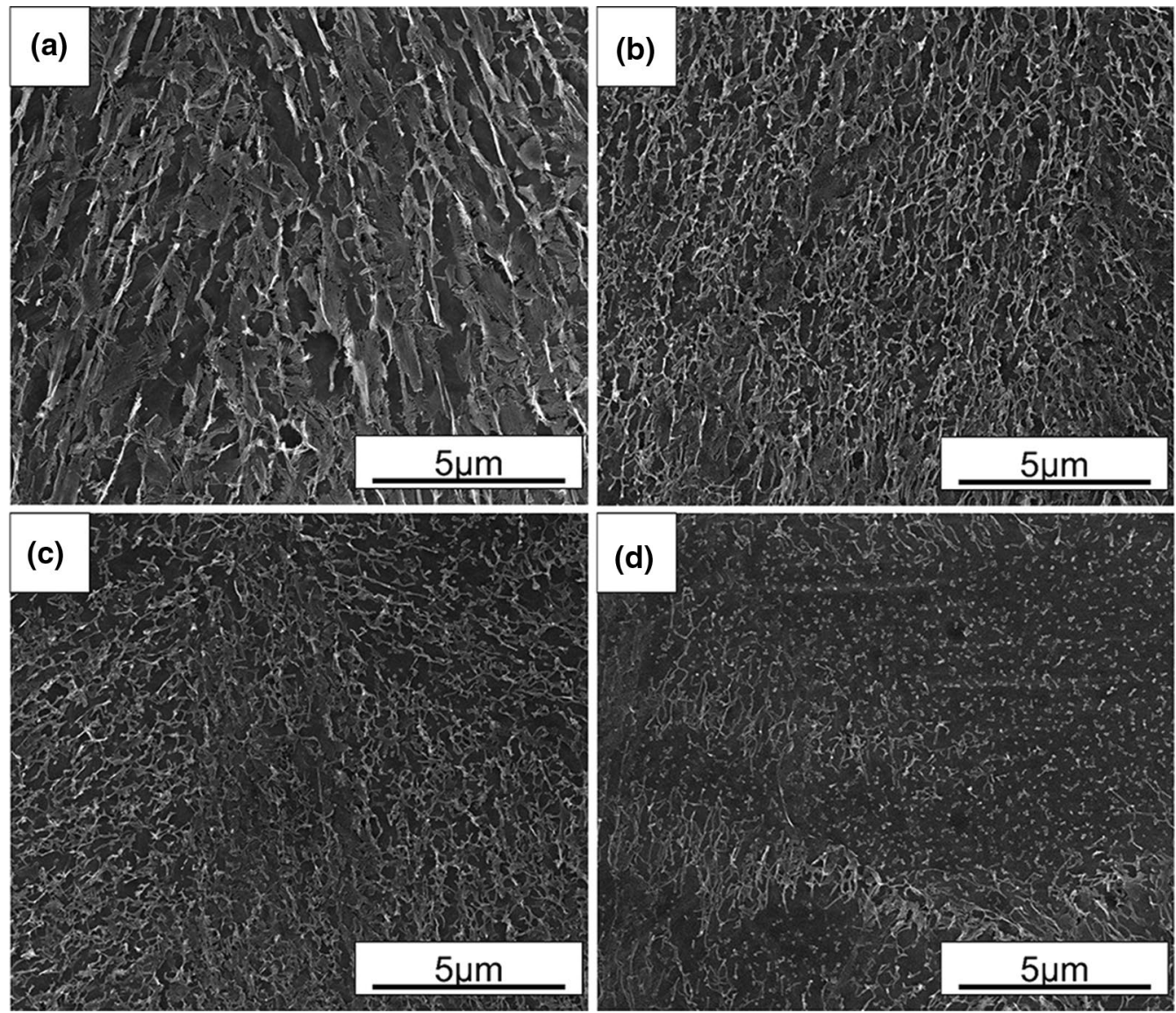

Fig. 12 SEM micrographs showing the microstructures of the as-built samples with $1 \mathrm{wt} \% \mathrm{TiB}_{2}$ on the longitudinal section at varied energy densities: a $416.67 \mathrm{~J} / \mathrm{mm}^{3}$; b $250 \mathrm{~J} / \mathrm{mm}^{3} ; \mathbf{c} 156.25 \mathrm{~J} / \mathrm{mm}^{3} ; \mathbf{d} 104.17 \mathrm{~J} / \mathrm{mm}^{3}$ 


\subsection{Mechanical Properties}

\subsubsection{Microhardness}

Figure 13 depicts the variation of microhardness of the asbuilt TiB/Ti6Al4V composites added with different contents of $\mathrm{TiB}_{2}$ at various energy densities. Apparently, the microhardness is influenced by both the content of $\mathrm{TiB}_{2}$ and the applied energy density. With the addition of $\mathrm{TiB}_{2}$, the maximum values of microhardness are increased from 440.765 to $468.752 \mathrm{Hv}$, and finally to $524.387 \mathrm{Hv}$ in the Ti6Al4V, $1 \mathrm{wt} \% \mathrm{TiB} / \mathrm{Ti} 6 \mathrm{Al} 4 \mathrm{~V}$ and $3 \mathrm{wt} \% \mathrm{TiB} / \mathrm{Ti} 6 \mathrm{Al} 4 \mathrm{~V}$ composites, respectively. However, as the energy density increases, the variations of microhardness of $\mathrm{TiB} / \mathrm{Ti} 6 \mathrm{Al} 4 \mathrm{~V}$ composites exhibit a different trend as compared to that of Ti6Al4V. The values of microhardness for the Ti6Al4V samples decrease with the decrease in energy density, which agrees well with the reported result in Ref. [42]. However, the values of microhardness for the TiB/Ti6Al4V composite firstly increase and then decrease as the energy density is decreased from 416.67 to $104.17 \mathrm{~J} / \mathrm{mm}^{3}$.

Generally, the microhardness is directly related to the densification level. In other words, a higher densification level usually means a higher resistance to the plastic deformation, thus a higher microhardness. Also, the finer the grains, the higher microhardness it possesses. As for the Ti6Al4V samples, the synergy effect of density and grain size decides the value of microhardness. It is worth noting that, due to more freedom for plastic deformation, the irregular defects caused by insufficient fusion more easily lead to the decline of microhardness as compared to the spherical pores. Thus, with the decrease in energy density, the weakening effect on microhardness caused by the declined density surpasses the enhancement induced by the refinement of grains, thus leading to a decrease in microhardness. For the TiB/Ti6Al4V samples, in addition to the effects of density and grain size, the TiB-reinforced phase

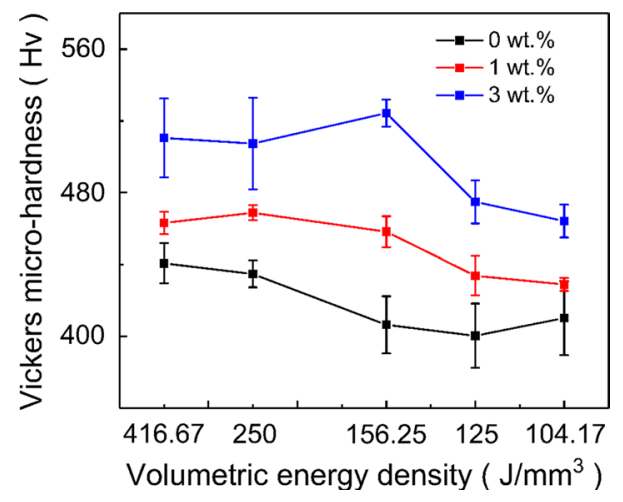

Fig. 13 Vickers microhardness of the as-built samples with different additions of $\mathrm{TiB}_{2}$ at different energy densities and its distribution also decide the value of microhardness. The introduction of the TiB-reinforced phase contributes to the increase of microhardness. Also, the more homogeneous distribution of the TiB-reinforced phase gives rise to a higher microhardness. Because the TiB/Ti6Al4V composites possess the densities of all above $97 \%$ with a uniform distribution of spherical pores, the effect of density on the microhardness of the TiB/Ti6Al4V samples can be ignored. For a given content of $\mathrm{TiB}_{2}$, when the energy density is in a relatively high range, the $\mathrm{TiB}$ phase distributes uniformly in the Ti6Al4V matrix due to the full fusion and reaction of $\mathrm{TiB}_{2}$ particles. At this moment, the effect of grain size plays a pivotal role in determining the microhardness. Thus, as the grains are refined with the decrease in energy density (still in a relatively high range), the microhardness consequently becomes higher. However, as the energy density is further decreased, the distribution of $\mathrm{TiB}$ becomes heterogeneous simultaneously. Both the grain size and the heterogeneous distribution of TiB affect the microhardness collectively. When the applied energy density is low enough, the effect of heterogeneous distribution of TiB dominates. That is to say, the weakening effect caused by the non-uniform distribution of $\mathrm{TiB}$ exceeds the enhancement effect resulted from the refinement of grains, thus the microhardness begins to decline. As a consequence, the variation trends of the microhardness with the energy density in the samples with different $\mathrm{TiB}_{2}$ contents are distinguishing (Fig. 13). With the decrease in energy density, the microhardness decreases due to the deterioration of the density in the Ti6Al4V samples, but increases firstly and then decreases in the TiB/ Ti6Al4V composites because of the synthetic effect of the grain size and TiB-reinforced phase. As the synthetic effect is partially determined by the content of $\mathrm{TiB}_{2}$, the maximum value of the microhardness $(468.752 \mathrm{Hv})$ is obtained at the energy density of $156.25 \mathrm{~J} / \mathrm{mm}^{3}$ in the $1 \mathrm{wt} \% \mathrm{TiB} / \mathrm{Ti} 6 \mathrm{Al} 4 \mathrm{~V}$ composites, while a higher maximum value $(524.387 \mathrm{Hv})$ is achieved at the energy density of $250 \mathrm{~J} / \mathrm{mm}^{3}$ in the $3 \mathrm{wt} \%$ TiB/Ti6Al4V composites.

\subsubsection{Friction and Wear Properties}

Figure 14 displays the friction coefficient curves of the asbuilt TiB/Ti6Al4V composites added with different contents of $\mathrm{TiB}_{2}$ at the energy density of $416.67 \mathrm{~J} / \mathrm{mm}^{3}$. It is remarkable that the friction behavior is significantly affected by $\mathrm{TiB}_{2}$ content, and the wear resistance is effectively enhanced with the increase in $\mathrm{TiB}_{2}$ addition. The friction coefficient of the Ti6Al4V sample with a mean value of 0.44 presents large fluctuations during the sliding process, indicating an unsteady sliding process. While the friction coefficients of the $1 \mathrm{wt} \%$ and $3 \mathrm{wt} \% \mathrm{TiB} / \mathrm{Ti} 6 \mathrm{Al} 4 \mathrm{~V}$ composites show no obviously large fluctuations and present a mean value of 0.38 and 0.35 , respectively. Note that the wear resistance is 


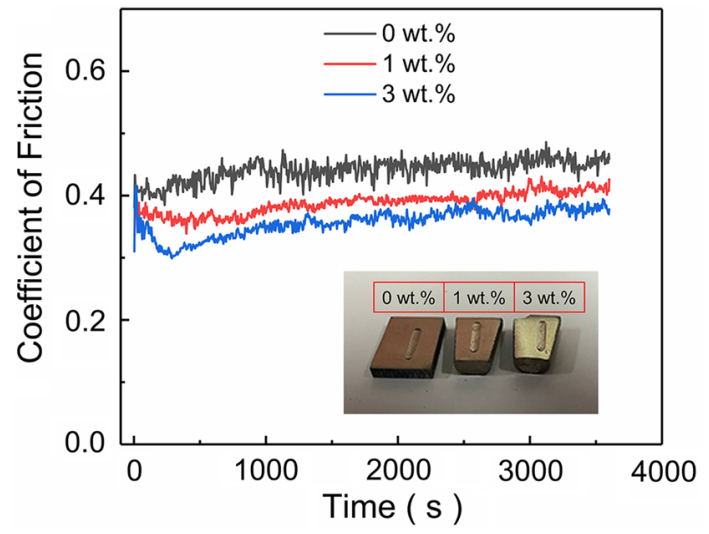

Fig. 14 Variation of friction coefficient with different additions of $\mathrm{TiB}_{2}$

enhanced accompanied by the increase in microhardness. Consequently, the wear resistance is mainly determined by the microhardness, which is also proved by Sulima et al. [43].

To investigate the wear mechanisms of various samples, the worn surface morphologies of the Ti6Al4V, $1 \mathrm{wt} \%$ and $3 \mathrm{wt} \% \mathrm{TiB} / \mathrm{Ti} 6 \mathrm{Al} 4 \mathrm{~V}$ composites were observed, as shown in Fig. 15. All the samples present obvious wear traces. The wear traces of TiB/Ti6Al4V composites present clearer and smoother surfaces compared to that of Ti6Al4V sample. SEM images with higher magnification are shown in Fig. 15d-f. A large amount of wear debris is clearly presented on the worn surface of the Ti6Al4V sample, only a little wear debris is found on the worn surfaces of TiB/Ti6Al4V composites. Delamination is only observed on the surface of TiB/Ti6Al4V composites. The grooves along the sliding direction also show different morphologies, which are discontinuous and deep in the Ti6Al4V sample but continuous and slight in the TiB/ Ti6Al4V composites. Thus, it can be concluded that the abrasive wear mechanism is changed from three-body to two-body with the addition of $\mathrm{TiB}_{2}$ [44]. For the Ti6Al4V sample, the three-body abrasive wear mechanism dominates due to the low microhardness. During the friction process, severe plastic deformation and repeated wear behavior proceed simultaneously, promoting the soft Ti6Al4V debris to be peeled from the worn surface. The Ti6Al4V debris will slide along with the $\mathrm{Si}_{3} \mathrm{~N}_{4}$ ball, thus the deep and long grooves are formed on the worn surface. For the TiB/Ti6Al4V composites, the two-body abrasive wear mechanism works. The hard and brittle TiB phases together with the refined microstructures contribute to the increase in microhardness, which significantly resists the deformation of the worn surface. The TiB/Ti6Al4V debris is harder to be peeled off and the amount of wear debris is reduced. Therefore, the wear resistance is effectively enhanced. However, due to the relatively low density, certain weak areas near pores on the surface of TiB/ Ti6Al4V composites are sheared and then ruptured under the repeated wear behavior, generating large-sized delamination. The higher wear resistance of $3 \mathrm{wt} \% \mathrm{TiB} / \mathrm{Ti} 6 \mathrm{Al} 4 \mathrm{~V}$
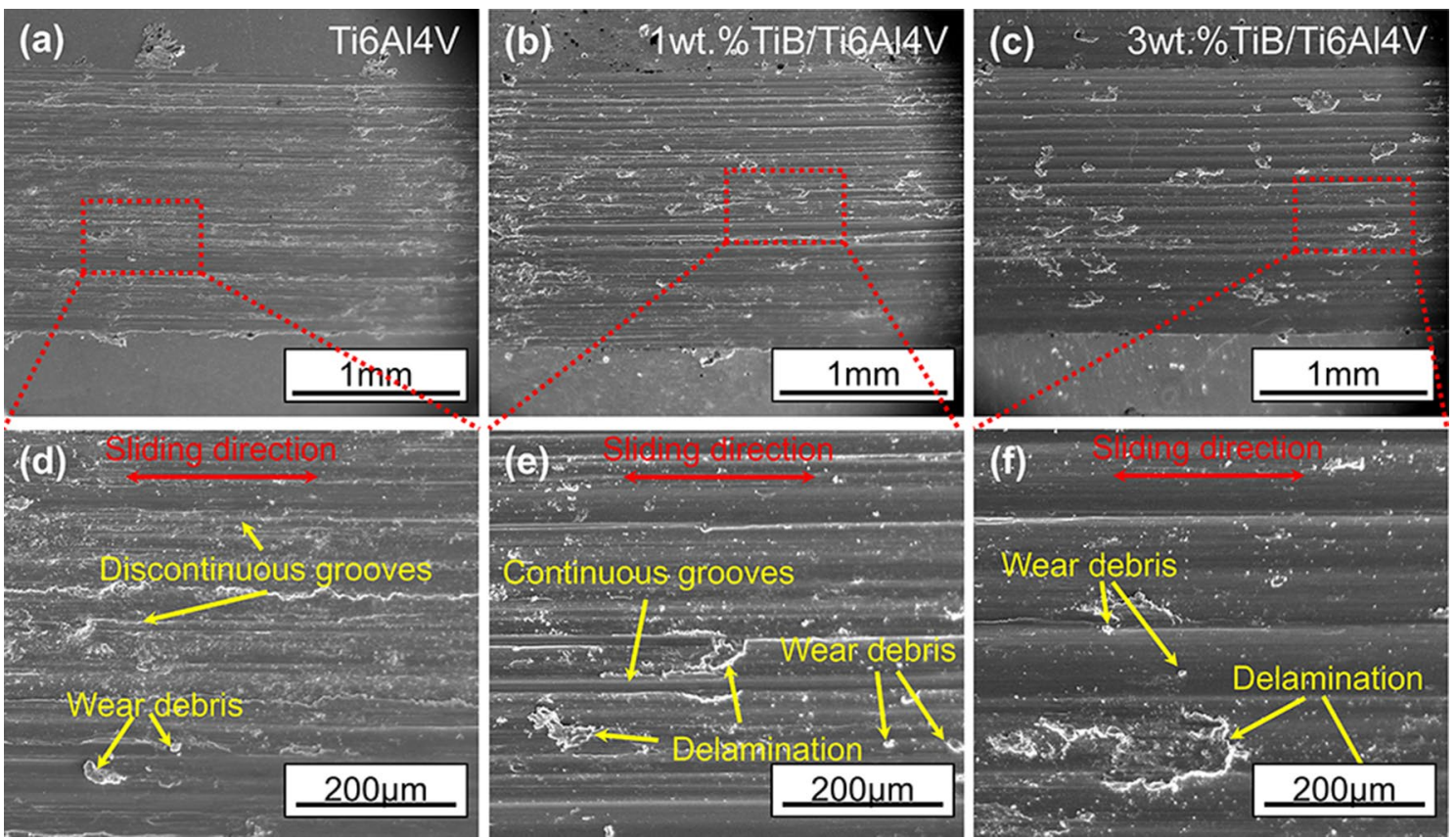

Fig. 15 SEM morphologies of the worn surfaces for the as-built samples: a, d Ti6Al4V; b, e 1 wt\% TiB/Ti6Al4V; c, f 3 wt\% TiB/Ti6Al4V 
composite is mainly ascribed to the higher microhardness compared to that of $1 \mathrm{wt} \% \mathrm{TiB} / \mathrm{Ti}$ Al4V composite.

\section{Conclusion}

In summary, the TiB/Ti6Al4V composites added with different contents of $\mathrm{TiB}_{2}$ are successfully in situ fabricated by SLM. The critical values of energy density for the good formability of samples added with $0 \mathrm{wt} \%, 1 \mathrm{wt} \%$ and $3 \mathrm{wt} \% \mathrm{TiB}_{2}$ are, respectively, at $125 \mathrm{~J} / \mathrm{mm}^{3}, 250 \mathrm{~J} / \mathrm{mm}^{3}$ and $416.67 \mathrm{~J} / \mathrm{mm}^{3}$. Cracks are found to originate from the edge of samples and end in the arc-shaped terminal. Moreover, reducing energy density can prevent the evaporation of $\mathrm{Al}$ with low melting points for the TiB/Ti6Al4V composites, thus contributing to the relatively high densities. Such rule is contrary to that of the $\mathrm{TiB}_{2}$-free samples. Additionally, the $\mathrm{TiB}$ phase is formed through the in situ reaction described as $\mathrm{Ti}+\mathrm{TiB}_{2} \rightarrow 2 \mathrm{TiB}$. The similarity of the lattice structures between the TiB and $\beta$-Ti facilitates the nucleation of $\mathrm{TiB}$ crystal adjacent to the $\beta$-Ti phase. Also, the needle-shaped TiB phase precipitates out along the boundaries of $\beta$-Ti grains and exhibits preferential growth along the [010] orientation, thus forming a basket-like microstructure. Moreover, with the decrease in energy density, the cooling rate significantly increases, which prevents the columnar crystals from growing along the preferential orientation. Consequently, the grains are refined. Meanwhile, the TiB phase transforms into a dot-like particle and its distribution becomes heterogeneous. These factors also reveal the change rule of microhardness with energy density for the TiB/Ti6Al4V composites. The microhardness and wear resistance of the TiB/Ti6Al4V composites are obviously improved as the content of $\mathrm{TiB}_{2}$ is increased from 0 to $3 \mathrm{wt} \%$. The abrasive wear mechanism is changed from three-body to two-body with the addition of $\mathrm{TiB}_{2}$. This study demonstrates the potential of developing the TiB/Ti6Al4V composites with an excellent performance by additive manufacturing technology for advanced applications.

Acknowledgements This work was financially supported by the Civil Aerospace Pre-research Project: research on additive manufacturing of core components in the liquid rocket engine, and the Fundamental Research Funds for the Central Universities (Nos. 2019kfyXMPY005 and 2019kfyXKJC042). The authors thank the Analytical and Testing Center and the State Key Laboratory of Materials Processing and Die \& Mould Technology of Huazhong University of Science and Technology for tests.

\section{References}

1. D.W. Ao, X.R. Chu, S.X. Lin, Y. Yang, J. Gao, Acta Metall. Sin. (Engl. Lett.) 31, 1287 (2018)
2. Y. Han, H.R. Wang, Y.D. Cao, W.T. Hou, S.J. Li, Acta Metall. Sin. (Engl. Lett.) 32, 1007 (2019)

3. C. Cui, B.M. Hu, L. Zhao, S. Liu, Mater. Des. 32, 1684 (2011)

4. F. Li, Z. Wang, X. Zeng, Mater. Lett. 199, 79 (2017)

5. J. Wang, X. Guo, L. Xiao, L. Wang, W. Lu, B. Li, Z. Li, D. Zhang, Acta Metall. Sin. (Engl. Lett.) 27, 205 (2014)

6. L. Geng, L. Huang, Acta Metall. Sin. (Engl. Lett.) 27, 787 (2014)

7. A.S. Patil, V.D. Hiwarkar, P.K. Verma, R.K. Khatirkar, J. Alloys Compd. 777, 165 (2019)

8. H. Attar, M. Bönisch, M. Calin, L.C. Zhang, S. Scudino, J. Eckert, Acta Mater. 76, 13 (2014)

9. M. Xia, A. Liu, Z. Hou, N. Li, Z. Chen, H. Ding, J. Alloys Compd. 728, 436 (2017)

10. M. Calin, A. Gebert, A.C. Ghinea, P.F. Gostin, S. Abdi, C. Mickel, J. Eckert, Mater. Sci. Eng. C 33, 875 (2013)

11. C. Cai, B. Song, C. Qiu, L. Li, P. Xue, Q. Wei, J. Zhou, H. Nan, H. Chen, Y. Shi, J. Alloys Compd. 710, 364 (2017)

12. L. Chen, Y. Yao, Acta Metall. Sin. (Engl. Lett.) 27, 762 (2014)

13. D. Zhou, F. Qiu, H. Wang, Q. Jiang, Acta Metall. Sin. (Engl. Lett.) 27, 798 (2014)

14. M. Moradi, M. Moazeni, H.R. Salimijazi, Vacuum 107, 34 (2014)

15. N. Kang, P. Coddet, Q. Liu, H.L. Liao, C. Coddet, Addit. Manuf. 11, 1 (2016)

16. P. Chui, Vacuum 154, 25 (2018)

17. D. Xu, W.J. Lu, Z.F. Yang, J.N. Qin, D. Zhang, J. Alloys Compd. 400, 216 (2005)

18. S.W. Maseko, A.P.I. Popoola, O.S.I. Fayomi, Def. Technol. 14, 408 (2018)

19. J.H. Tan, W.L.E. Wong, K.W. Dalgarno, Addit. Manuf. 18, 228 (2017)

20. S. Luo, P. Gao, H. Yu, J. Yang, Z. Wang, X. Zeng, J. Alloys Compd. 771, 387 (2019)

21. S. Luo, C. Zhao, Y. Su, Q. Liu, Z. Wang, Addit. Manuf. 31, 100925 (2020)

22. Y. Zhang, A. Bandyopadhyay, Addit. Manuf. 29, 100783 (2019)

23. X. Yan, Q. Li, S. Yin, Z. Chen, R. Jenkins, C. Chen, J. Wang, W. Ma, R. Bolot, R. Lupoi, Z. Ren, H. Liao, M. Liu, J. Alloys Compd. 782, 209 (2019)

24. L.Z. Wang, W.H. Wei, Acta Metall. Sin. (Engl. Lett.) 31, 807 (2018)

25. P. Hu, Y.H. Zhou, J. Deng, S.L. Li, W.J. Chen, T. Chang, B.L. Hu, K.S. Wang, P.F. Feng, A.A. Volinsky, J. Alloys Compd. 745, 532 (2018)

26. N.A. Rubinkovskii, D.P. Shornikov, A.V. Tenishev, A.G. Zaluzhnyi, A.G. Zholnin, Glas. Ceram. -Engl. Transl. Steklo I Keramika 76, 27 (2019)

27. H. Shipley, D. McDonnell, M. Culleton, R. Coull, R. Lupoi, G. O’Donnell, D. Trimble, Int. J. Mach. Tools Manuf. 128, 1 (2018)

28. P. Petrov, C. Georgiev, G. Petrov, Vacuum 51, 339 (1998)

29. D. Gu, Y. Yang, L. Xi, J. Yang, M. Xia, Opt. Laser Technol. 119, 105600 (2019)

30. P. Tan, F. Shen, B. Li, K. Zhou, Mater. Des. 168, 107642 (2019)

31. Z.Y. Hu, X.W. Cheng, S.L. Li, H.M. Zhang, H. Wang, Z.H. Zhang, F.C. Wang, J. Alloys Compd. 726, 240 (2017)

32. X. Ma, C. Li, K. Bai, P. Wu, W. Zhang, J. Alloys Compd. 373, 194 (2004)

33. B.G. Fu, H.W. Wang, C.M. Zou, Z.J. Wei, Trans. Nonferrous Met. Soc. China (Engl. Ed.) 25, 2206 (2015)

34. L.J. Huang, L. Geng, H.X. Peng, J. Zhang, Scr. Mater. 64, 844 (2011)

35. B. Zhou, J. Zhou, H. Li, F. Lin, Mater. Sci. Eng. A 724, 1 (2018)

36. P. Nandwana, N. Gupta, S.G. Srinivasan, R. Banerjee, Comput. Mater. Sci. 150, 197 (2018)

37. Y. Tian, N. Chekir, X. Wang, A. Nommeots-Nomm, R. Gauvin, M. Brochu, Addit. Manuf. 24, 137 (2018)

38. A. Rai, H. Helmer, C. Körner, Addit. Manuf. 13, 124 (2017) 
39. J. Yin, H. Zhu, L. Ke, W. Lei, C. Dai, D. Zuo, Comput. Mater. Sci. 53, 333 (2012)

40. L.J. Huang, L. Geng, B. Wang, L.Z. Wu, Mater. Des. 45, 532 (2013)

41. A.F. Manchón-Gordón, J.S. Blázquez, C.F. Conde, A. Conde, J. Alloys Compd. 675, 81 (2016)
42. J. Han, J. Yang, H. Yu, J. Yin, M. Gao, Z. Wang, X. Zeng, Rapid Prototyp. J. 23, 217 (2017)

43. I. Sulima, P. Klimczyk, P. Malczewski, Acta Metall. Sin. (Engl. Lett.) 27, 12 (2014)

44. N. Kang, W. Ma, F. Li, H. Liao, M. Liu, C. Coddet, Vacuum 154, 69 (2018) 